\title{
Die Medialität des Theaters bei Frank Wedekind
}

Eine medientheoretische Untersuchung über den Einfluss des Bänkelsängers und Schauspielers Frank Wedekind auf sein Werk 
Die vorliegende Arbeit wurde vom Fachbereich 13 - Neuphilologie der Johannes Gutenberg-Universität Mainz im Jahr 2004 als Dissertation zur Erlangung des akademischen Grades eines Doktors der Philosophie (Dr. phil.) angenommen. 
Referent:

Prof. Dr. Andreas Solbach

Korreferentin:

Prof. Dr. Ariane Martin

Tag des Prüfungskolloquiums: $\quad 30$. August 2004 


\section{Reihe Sprachwissenschaft}

früher Reihe Sprach- und Literaturwissenschaft

Band 37 


\section{Die Medialität des Theaters bei Frank Wedekind}

Eine medientheoretische Untersuchung über den Einfluss des Bänkelsängers und Schauspielers Frank Wedekind auf sein Werk

\section{Georg W. Forcht}

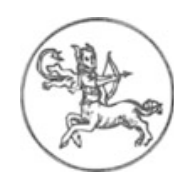

Centaurus Verlag \& Media UG 2005 
Georg W. Forcht, geb. 1943, Oberstudienrat, absolvierte ein Studium für das Lehramt an Volksschulen sowie ein Studium für Hörgeschädigtenpädagogik. Er war an der Berufsschule für Hörgeschädigte in Frankenthal tätig, die er 1974 aufgebaut hatte und zu zu deren Leiter er 1981 ernannt wurde. 1995 begann er in Mainz das Studium der Deutschen Philologie. Er legte 1997 das 1. und 2. Staatsexamen für das Lehramt im höheren Dienst an berufsbildenden Schulen ab und promovierte 2004 am Fachbereich Neuphilologie der Universität Mainz.

\section{Die Deutsche Bibliothek - CIP-Einheitsaufnahme}

\section{Forcht, Georg W.:}

Die Medialität des Theaters bei Frank Wedekind :

Eine medientheoretische Untersuchung über den Einfluss

des Bänkelsängers und Schauspielers Frank Wedekind

auf sein Werk / Georg W. Forcht. - Herbolzheim : Centaurus-Verl., 2005

(Reihe Sprach- und Literaturwissenschaft ; Bd. 37)

Zugl.: Mainz, Univ., Diss., 2004

$$
\text { ISBN 978-3-8255-0529-5 ISBN 978-3-86226-368-4 (eBook) }
$$

DOI 10.1007/978-3-86226-368-4

\section{ISSN 0177-2821}

Alle Rechte, insbesondere das Recht der Vervielfältigung und Verbreitung sowie der Übersetzung, vorbehalten. Kein Teil des Werkes darf in irgendeiner Form (durch Fotokopie, Mikrofilm oder ein anderes Verfahren) ohne schriftliche Genehmigung des Verlages reproduziert oder unter Verwendung elektronischer Systeme verarbeitet, vervielfältigt oder verbreitet werden.

(C) CENTAURUS Verlags-Gmbh \& Co. KG, Herbolzheim 2005

Satz: Vorlage des Autors

Umschlagabbildung: Briefumschlag an den „Thierbändiger“ Frank Wedekind. Die Abbildungen wurden zur Verfügung gestellt vom Historischen Museum Aargau (Kantonale Sammlungen, Schloss Lenzburg), Schweiz. 
Besonderer Dank gilt meinem Doktorvater Herrn Prof. Dr. Andreas Solbach für seine große Unterstützung und seine wertvollen Anregungen, die zum Gelingen der Arbeit wesentlich beigetragen haben, sowie für die Anfertigung seines Erstgutachtens. Auch danke ich für die mir während der gesamten Bearbeitungsphase entgegengebrachte menschliche Wärme.

Frau Prof. Dr. Ariane Martin danke ich für ihre Bereitschaft, das Zweitgutachten zu übernehmen.

$\mathrm{Zu}$ aufrichtigem Dank verpflichtet bin ich auch meinem früheren Lehrer, Herrn Prof. Dr. Dieter Kafitz, der mir in der Vorbereitungsphase zu meiner Dissertation seinen persönlichen Rat und sein Wohlwollen schenkte.

Eine wertvolle Hilfe waren auch die Damen und Herren aus den Archiven und Bibliotheken. Stellvertretend für alle seien hier Herr Dönni von der Kantonsbibliothek Aarau, Frau Dr. Ball vom Historischen Museum Schloss Lenzburg, Herr Huber vom Burghaldemuseum Lenzburg und Frau Hummel von der Stadtbibliothek München genannt. 


\section{Vorwort}

Die Forschung zu dem literarischen Werk Frank Wedekinds bietet ein uneinheitliches Bild, zu dem nicht nur der frühe Tod des Autors, sondern auch die traditionelle Rollenzuschreibung an einen Dichter, der sich als Rezitator, Bänkelsänger, Regisseur und Schauspieler seiner eigenen Stücke betätigte, beigetragen hat. Die Vielseitigkeit seines Werks, macht es somit erforderlich, einen neuen Ansatz zu suchen.

Die vorliegende Arbeit wendet sich der Analyse des Werks mit Hilfe feministischer und psychoanalytischer Modelle zu, so dass eine Mischung aus philologischer Praxis und analytischer Innovation entstanden ist, die neue Perspektiven eröffnen soll.

Der erste Teil der Arbeit widmet sich der Medialität des Theaters bei Frank Wedekind, in dem die Vorbilder für seine literarische Produktion in der Volksstücktradition, im Münchner Volkssängertum und in der Kabarettradition der Jahrhundertwende als prägende Faktoren beschrieben werden. Dabei arbeitet der Verfasser biographisch, indem er rekonstruierbare Quellen Nepomuk Nestroys oder Karl Valentins anführt und auf Übereinstimmung analysiert.

Der zweite Teil der Arbeit ist durch eine detaillierte Analyse der sechs bedeutendsten Dramen - Frühlings Erwachen, Der Marquis von Keith, Die 'Lulu'Dramen, Der Kammersänger, König Nicolo und Franziska - gekennzeichnet. Die Dramenanalysen folgen dem identischen Dreischritt von "Entstehungsgeschichte, Wirkung und Forschung", "Handlung" und "Analyse". Dabei versucht der Autor durch ständige Rückbezüge zur Volksstück- und Volkssängertradition bzw. zu den Bänkelsängern die Medialität des Theaters bei Wedekind nachzuzeichnen. 


\section{Inhaltsverzeichnis}

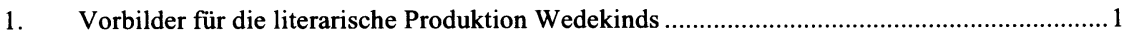

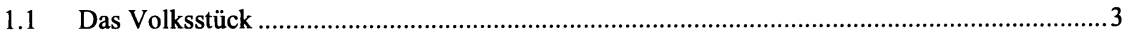

1.2. Die Vorläufer des Wiener Volksstücks ............................................................................

1.3. Die Volksstücktradition bei Johann Nepomuk Nestroy .........................................................

2. Beschreibung und Analyse des Münchner Volkssängertums um die Jahrhundertwende ........19

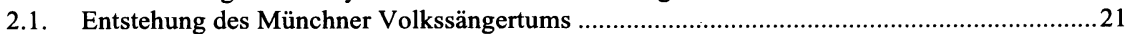

2.2. Figuren der Volkssängerdarstellungen ..............................................................................22

2.3. Künstlerische Formen des Volkssängertums .....................................................................2

2.4. Themen und Stoffe der Volkssänger ...................................................................................23

3. Die Bänkelsänger und Entertainer Karl Valentin und Frank Wedekind...................................26

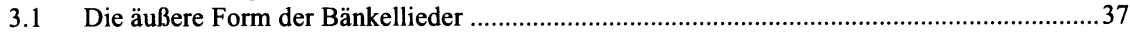

4. Die theatralen Mittel bei Karl Valentin und Frank Wedekind ................................................44

4.1. Zeitgeschichtliche und expressionistische Einflüsse auf die Autoren .....................................44

4.2. Die Identifikation von Person und Schauspieler ....................................................................46

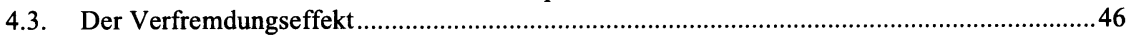

5. Traditionelle und neue Wege der Bühnen- und Schauspielkunst ...........................................48

5.1. Die Bedeutung der dramatischen Figuren bei Wedekinds Inszenierungen ............................49

5.2. Wedekinds Musikverständnis.........................................................................................56

6. Dramenanalysen unter der besonderen Berücksichtigung der populären volkstheatralischen

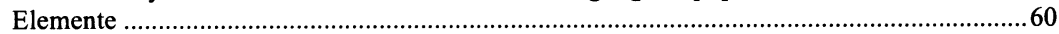

6.1. Frühlings Erwachen. Eine Kindertragödie ........................................................................61

6.1.1. Entstehungsgeschichte, Wirkung und Forschung ............................................................61

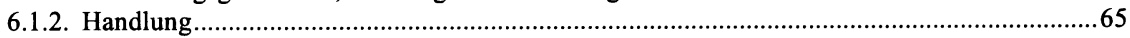

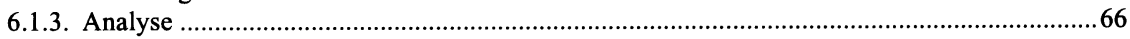

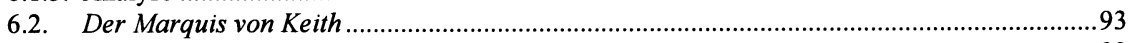

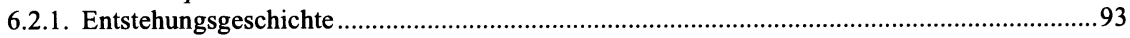

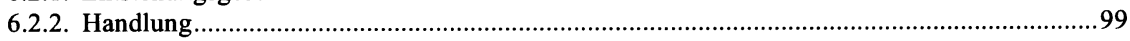

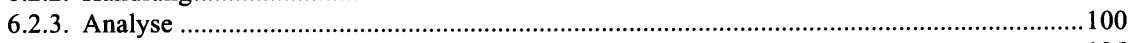

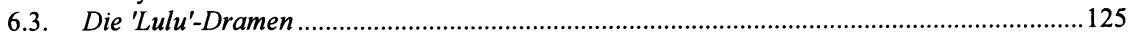

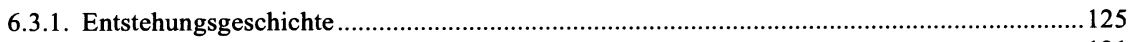

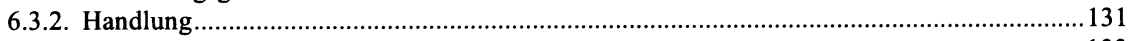

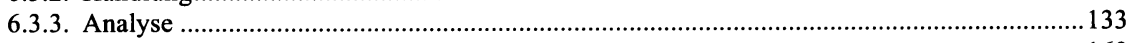

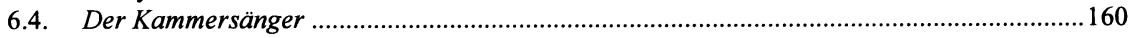

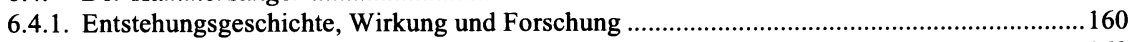

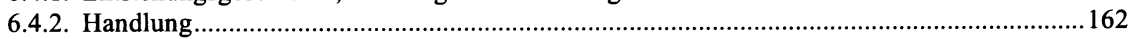

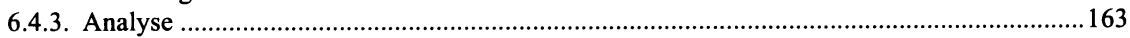

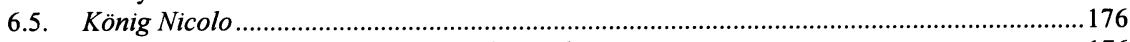

6.5.1. Entstehungsgeschichte, Wirkung und Forschung ...........................................................176

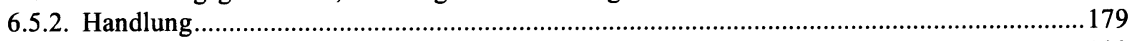

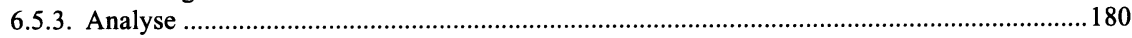

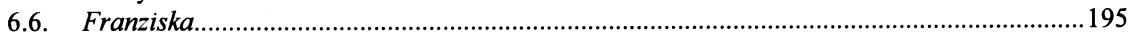

6.6.1. Entstehungsgeschichte, Wirkung und Forschung ..........................................................195

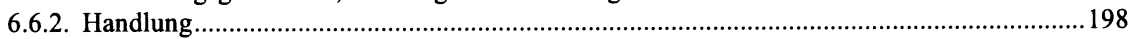

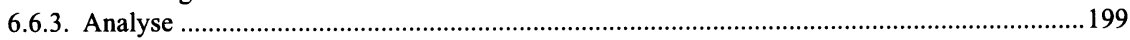

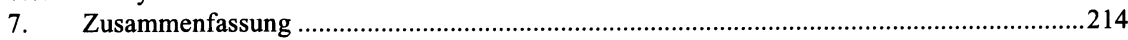

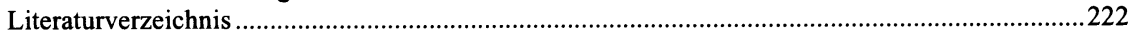




\section{Siglenverzeichnis}

GW: Wedekind, Frank: Gesammelte Werke. 9 Bde. München: Müller, 1924

GB: Wedekind, Frank: Gesammelte Briefe. 2 Bde. Hg. Fritz Strich. München, 1924

T: Wedekind, Frank: Die Tagebücher. Hg. Gerhard Hay. Frankfurt a.M. 1986

$\mathrm{Nb}$ : Notizbücher. Wedekind-Archiv München. Monacensia Literaturarchiv

Die Notizbücher und ein Konvolut loser Notizblätter sind in der Handschriftenabteilung unter L 3501/1-67 registriert. Das Rekto des jeweiligen Blattes wird mit „R“, das Verso mit „V“ kenntlich gemacht

Zum Beispiel bedeutet Nb. 1/1R: Notizbuch 1, erstes Blatt, Rekto

TbF: Tagebuch Friedrich Wilhelm Wedekind in französischer Sprache

Münchner Stadtbibliothek. Monacensia Literaturarchiv L 3476/46

JE: Jugenderinnerungen Emilie Wedekind-Kammerer. Münchner Stadtbibliothek Monacensia Literaturarchiv L 3476/45

RB: Regiebücher. Münchner Stadtbibliothek. Monacensia Literaturarchiv

BML: Burghaldemuseum, Lenzburg

\section{Bemerkungen}

Die Jahreszahl in Klammer, nach dem Titel des Dramas, bezieht sich auf das jeweilige Erscheinungsjahr.

\section{Archive}

Aargauische Kantonsbibliothek:

Kantonsbibliothek Aarau, Aargauerplatz 1, CH-5001 Aarau

Wedekind Archiv Lenzburg:

Burghaldemuseum, Schlossgasse 23, CH-5600 Lenzburg

Wedekind Archiv Lenzburg:

Historisches Museum Schloss Lenzburg, CH-5600 Lenzburg

Wedekind-Archiv München:

Stadtbibliothek München, Monacensia-Abteilung, Maria Theresia Strasse 23, D-81675

München/Bogenhausen

Frank Wedekind - Gesellschaft e.V. Darmstadt

Haardtring 100, D-64295 Darmstadt

Österreichische Nationalbibliothek:

Zeitungsarchiv, Josefplatz 1, A-1015 Wien 